\title{
Research of the hydraulic resistance of the granular filter of the sweeping mashnine
}

\author{
Rashid Sharapov ${ }^{1 *}$ \\ ${ }^{1}$ Moscow State University of Civil Engineering, 129337, 26, Yaroslavskoye Shosse, Moscow, Russia
}

\begin{abstract}
The article focuses on the need to improve the ecology in modern cities based on the development of new cleaning equipment. It is proposed to use a granular filter as a dust cleaning unit in sweeping machines. The advantages of granular filters over other types of filtering apparatus are given. The basic schemes of working equipment of sweepers are considered. To describe the processes during air purification in the proposed granular filter, mathematical expressions are proposed to determine the hydraulic resistance of the proposed filter. When developing analytical expressions, the structural and technological features of the proposed filter were taken into account. The results of calculating the hydraulic resistance of the proposed granular filter depending on its main structural and technological parameters are obtained. To confirm the obtained analytical expressions, experimental studies were carried out. The clinker of the Belgorod cement plant of a fraction of $-10+5 \mathrm{~mm}$ was selected as the filtering material. During the experiment, cement was used as dust with a specific surface of various dispersion, determined by the parameter $\delta_{50}$. In addition, filtering speed and filtering time are accepted as variable parameters.
\end{abstract}

\section{Introduction}

The development of modern systems and equipment, as well as the desire of modern society to improve the cleanliness of the road surface and the air in residential areas and industrial premises, lead to the actualization of the development and improvement of machines for cleaning dusty air [1-5].

One of the central mechanisms of the sweeper, which has a significant impact on the parameters of the air that comes out after suction along with dust and debris inside the machine, is air purification filters.

One of the key features of granular filters is the ability to work at high (up to $700{ }^{\circ} \mathrm{C}$ ) temperatures, aggressive environments and very abrasive dust [6-8]. At the same time, very effective regeneration is carried out in granular filters while preserving the filter material itself.

Gas purification from dust and other physical contaminants in these filters is carried out by passing it through bulk, up to 100 ... 300 millimeters high, a layer of granules whose size is from 2 to 10 millimeters. The regeneration of the filtering layer is most often carried out

* Corresponding author: ptdm_zavkaf@mail.ru 
by mechanical tedding in combination with reverse blowing. During reverse blowing, dusty air passes through the cyclone and mixes with the raw gas. The use of granular filters in the sweeping mashnines, in contrast to bag filters, can reduce costs, as the data show, by about $20 \ldots 25 \%$, due to their reliability and durability. The advantages of granular filters are that they are cheaper than bag filters, and therefore they pay off more quickly and provide a lower final filtering cost. However, until now, studies to determine the hydraulic resistance of these devices are not enough [9-12].

\section{Methods}

In this regard, the work on the design of the granular filter and the study of its hydraulic resistance can be considered an relevant scientific and practical task. The use of granular filters in the sweeping mashnines are due not only to their high performance and the ability to filter contaminants to a purity of $99.5 \%$, but also to the relative cheapness of operation of this type of filter. This reduces the cost of operating machines with this type of filter.

Vacuum sweepers are designed to clean city streets, including highways, as well as surrounding areas and perform work on cleaning various hard surfaces. They are equipped with various additional equipment and the result is a universal sweeper [13].

In fig. 1 presents the basic schemes of the working equipment of sweepers.

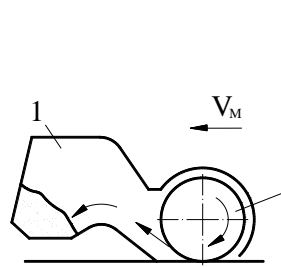

a)

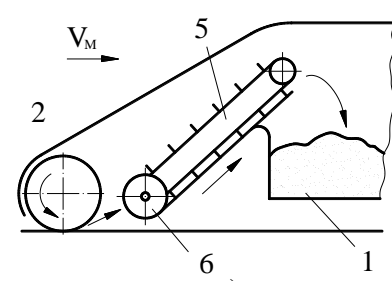

e)

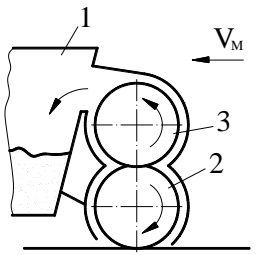

c)

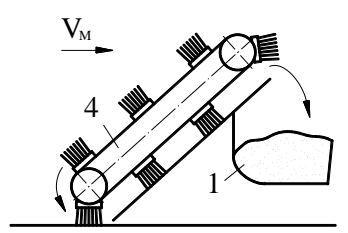

d)
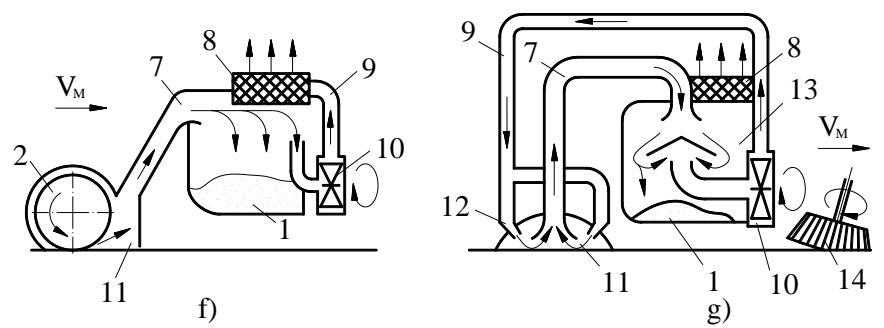

Fig. 1. Schemes of working equipment for sweepers: a - direct casting estimates; b - reverse casting estimate; $\mathrm{c}$ - casting estimates with a blade thrower; $\mathrm{g}$ - cast estimate with a brush; $\mathrm{d}$ - equipping with chain-scraper and screw conveyors; e - equipping with a brush-vacuum pick-up and gravity separation estimates; $\mathrm{g}$ - cost estimates equipped with a jet-vacuum pick-up and inertial compartment; 1 - hopper; 2 - the cylindrical brush; 3 - the blade thrower; 4 - the belt brush; 5 - the scraper conveyor; 6 - an auger; 7 - the suction pipe; 8 - a filter; 9 - the pressure pipeline; 10 - the vacuum fan; 11 - the vacuum pick-up; 12 - the blowing nozzles; 13 - a cyclone; 14 - the conical brush

To improve the degree of cleaning of city streets, courtyards and parks, we proposed a new design of a bulk rotating granular filter (Fig. 2).

The design of this filter is performed stepwise, with a decrease the filter layer, as the speed and volume of dusty gas decrease. This allows you to solve several design problems at once: uneven flow rate and reduce hydraulic resistance. The filter itself rotates around its horizontal axis. During operation, the filter layer is gradually clogged, thereby increasing the filtration efficiency, but the hydraulic resistance increases. With high hydraulic 
resistance, regeneration of the filter is necessary. The filter is rotary and when it is rotated and the clinker layers are poured out, regeneration occurs.
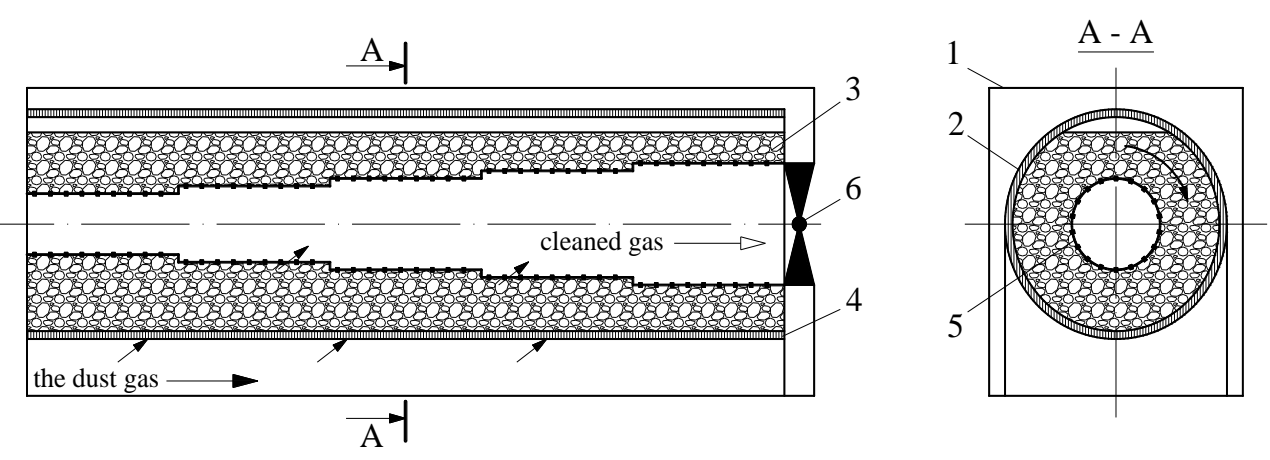

Fig. 2. Granular filter: 1 - housing; 2 - a drum; 3 - the filter layer; 4 - a lattice; 5 - nozzles; 6 - a fan

The quality of street cleaning and of air cleaning by sweeping machines during operation has a significant impact on the maximum permissible concentration of dust in the air and its chemical composition.

In our opinion, it is rational to use granular filters in sweeping machines by the criterion of cost and speed of recovery.

An analysis of literary sources has shown that most often studies relate to air purification in various industries than for use directly in sweeping equipment. Thus, we can conclude that it is advisable to conduct a study of granular filters for sweeping equipment for various characteristics to form the optimal filter design based on the tasks of cleaning the territory.

To describe the processes during air purification in a granular filter, it is advisable to consider the granular layer as an isotropic quasihomogeneous medium, which is characterized by some averaged parameters. First of all, they must include porosity, as well as the specific surface of the granular layer.

The expression for determining the porosity of the granular layer:

$$
\varepsilon=1-\frac{\rho_{b}}{\rho_{g}},
$$

where $\rho_{b}$ is the bulk density of the layer as a whole, $\mathrm{kg} / \mathrm{m}^{3} ; \rho_{g}$ - granular density, $\mathrm{kg} / \mathrm{m}^{3}$.

The volume fraction of a solid skeletal filter layer is called its packing density $\alpha$ and is determined by the formula:

$$
\alpha=1-\varepsilon=\frac{\rho_{b}}{\rho_{g}} .
$$

If the grains of the bulk layer have an internal porosity $\varepsilon_{i}$, then we can conclude that $\rho_{g}$ must be understood as the apparent density $\rho_{a}$, which can be determined from the formula:

$$
\rho_{a}=\frac{m_{g}}{V_{g}}=\left(1-\varepsilon_{i}\right) \rho_{t},
$$


where $m_{g}$ is the mass of the granular layer, $\mathrm{kg} ; V_{g}$ - the volume of the granular layer, $\mathrm{m}^{3} ; \rho_{t}$ is the true density of the grain material, $\mathrm{kg} / \mathrm{m}^{3}$.

The dispersion of the granular layer is also characterized by a specific surface equal to the total surface of all grains per unit volume (or mass) of the layer according to the formula:

$$
S=S_{0}(1-\varepsilon) K_{n},
$$

where $\varepsilon$ is the porosity of the granular layer; $K_{n}<1$ is the screening coefficient of grains, which takes into account the decrease in the total surface of the grains of the layer due to their contact; $S_{0}$ is the specific surface area of the grain (average), which is determined by the formula:

$$
S_{0}=\frac{S_{g}}{m_{g 1}},
$$

where $S_{g}$ - the grain area, $\mathrm{m}^{2} ; m_{g 1}$ - the grain mass, $\mathrm{kg}$.

The efficiency of inertial deposition of particles in a layer of bulk material is characterized by the dimensionless criterion, the Stokes Number (Stk), which is defined as the ratio of the inertia to the aerodynamic drag of a gas according to the expression:

$$
\mathrm{Stk}=\frac{\delta \cdot \rho_{a} \cdot \mu}{18 d_{3}} K_{c},
$$

where $\delta$ is the size of the captured dust particles, $\mathrm{m} ; \rho_{b}$ is the volume of the filter bowl, $\mathrm{m}^{3}$; $\mu$ is the velocity of the dusty gas flow, $\mathrm{m} / \mathrm{min} ; d_{g}$ - grain diameter, $\mathrm{m} ; K_{c}$ is the Cunningham - Milliken Amendment [14], which takes into account an increase in the mobility of particles whose size is comparable to the mean free path of gas molecules, which is determined by the formula:

$$
K_{c}=1+\frac{2 \lambda}{\delta}\left(1.25+0,4 \exp \left(-0.55 \frac{\delta}{\lambda}\right)\right)
$$

where $\lambda$ is the mean free path of the molecules (at $t=20^{\circ} \mathrm{C}, P_{b}=1.01 \cdot 10^{5} \mathrm{~Pa}$, taken equal to $\left.\lambda=6.5 \cdot 10^{-8} \mathrm{~m}\right)$.

As the filtering material, we chose the clinker of the Belgorod cement plant with fraction $-10+5 \mathrm{~mm}$. During the experiment, cement with a different specific surface was used as dust. When performing experimental research studies to determine the hydraulic resistance of bulk filter layers, it was determined that a decrease in dust leakage through the layer, as well as an increase in hydraulic resistance of the filter, are two quantitative characteristics of the same process - namely, filling a dusty granular filter layer with dust caught. That is why, we can conclude that the main influencing factors and the dominant dimensionless criteria that determine the hydraulic resistance of the bulk layer remain the same as in the study of dust collection efficiency [15-17].

In general terms, the hydraulic resistance of a filter with a rotating granular layer is determined by the formula, $\mathrm{Pa}$ :

$$
\Delta \rho_{f}=\Delta \rho_{1}+\Delta \rho_{2}
$$

where $\Delta \rho 1$ is the resistance of the granular layer, $\mathrm{Pa} ; \Delta \rho 2$ is the resistance during the accumulation of captured dust, $\mathrm{Pa}$. 
The resistance of the granular layer is determined by the formula, $\mathrm{Pa}$ :

$$
\Delta \rho_{1}=\frac{13.13 \cdot(1-\varepsilon) \cdot w^{2} \cdot \rho \cdot H_{l}}{\varepsilon_{d}^{3} \cdot d_{e q}}
$$

where $w$ is the filtration rate, $\mathrm{m} / \mathrm{s} ; H_{l}$ - the height of the granular layer, $\mathrm{m} ; \varepsilon_{d}$-dust porosity, $\% ; d_{e q}$ is the equivalent diameter of the grain of the layer, $\mathrm{m}$.

The increase in resistance due to the accumulation of captured dust is determined by the formula, $\mathrm{Pa}$ :

$$
\Delta \rho_{2}=\frac{19.7 \cdot 10^{-5} \cdot w \cdot z_{1} \cdot t_{f} \cdot \varepsilon_{i}}{d_{e q}^{2} \cdot d_{f}^{1 / 2}}
$$

where $z_{1}$ is the initial dustiness of the gas, $\mathrm{kg} / \mathrm{m}^{3} ; t_{f}$ - filtering time, $\mathrm{s} ; \varepsilon_{i}$ - internal porosity of the grain layer, $\mathrm{kg} / \mathrm{m}^{3} ; d_{e q}$ - equivalent grain diameter of the layer, $\mathrm{m} ; d_{f}$ is the diameter of the filter, $\mathrm{m}$. With a stepwise diameter of the filter, it is taken as the average value of the corresponding diameters.

\section{Discussion}

Taking into account the results of various researchers [18-20], an intermediate formula for the hydraulic resistance of the granular filter was obtained:

$$
\Delta \rho_{f}=1686.7\left(\frac{d_{g}}{d_{g 0}}\right)^{-0.127} \cdot\left(\frac{w}{w_{0}}\right)^{0.132} \cdot\left(\frac{\delta_{50}}{\delta_{0}}\right)^{0.217} \cdot\left(\frac{z_{i}}{z_{i 0}}\right)^{0.161} \cdot\left(\frac{\tau}{\tau_{0}}\right)^{0.117}
$$

where $d_{g}$ - grain diameter, $\mathrm{m} ; d_{g 0}$ - specific grain diameter, $\mathrm{m} ; w$ is the initial filtration rate, $\mathrm{m} / \mathrm{s} ; w_{0}$ is the final filtration rate, $\mathrm{m} / \mathrm{s} ; \delta_{50}-$ the initial size of the captured dust particles, $\mathrm{m} ; \delta_{0}$ is the final size of the captured dust particles, $\mathrm{m} ; z_{i}$ - initial dust concentration, $\mathrm{kg} / \mathrm{m}^{3} ; z_{H 0}$ - final dust concentration, $\mathrm{kg} / \mathrm{m}^{3} ; \tau$ is the initial filtering time, $\mathrm{h} ; \tau_{0}$ is the final filtering time, $\mathrm{h}$.

From here formula of the hydraulic resistance of the granular layer of the developed filter was obtained:

$$
\Delta \rho_{f}=3773.4 \mathrm{Re}^{-0.094} \cdot \mathrm{Stk}^{0.109} \cdot H^{.0 .47} \cdot\left(\frac{z_{H}}{\rho}\right)^{0.192} \cdot\left(\frac{d_{k}}{h}\right)^{0.161},
$$

The dependence of the hydraulic resistance of the granular layer on the main influencing factors is presented in Figure 3. To confirm the obtained analytical expressions, an experimental setup was created and field tests were conducted. When conducting experimental studies, we varied: the specific grain diameter of the filter material, the filtration rate, the specific surface of dust with various dispersion, determined by the parameter $\delta 50$ and the filtration time. 


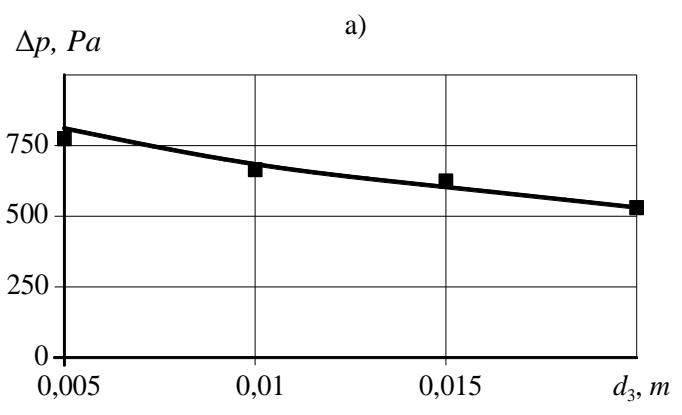

$\triangle p, P a$

c)

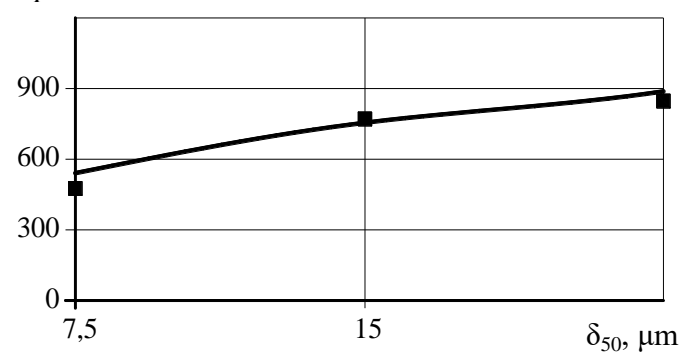

b)

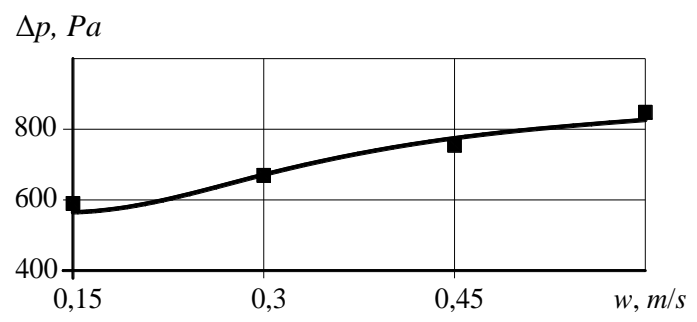

d)

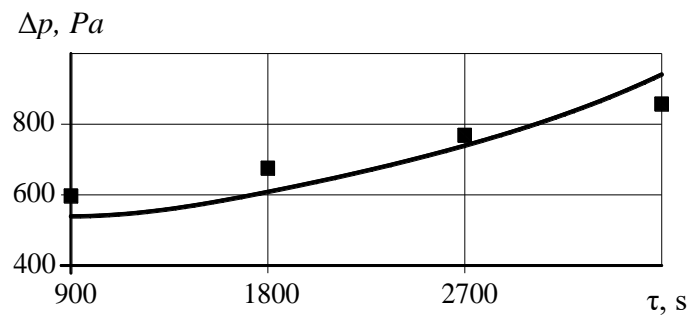

Fig. 3. The dependence of the hydraulic resistance of the granular layer on the main influencing factors

$a: w=0.3 \mathrm{~m} / \mathrm{s} ; \delta_{50}=15 \mu \mathrm{m} ; z_{\mathrm{H}}=0.01 \mathrm{~kg} / \mathrm{m}^{3} ; \tau=1800 \mathrm{~s} ;$

$b: \delta_{50}=15 \mu \mathrm{m} ; z_{\mathrm{H}}=0.01 \mathrm{~kg} / \mathrm{m}^{3} ; d_{3}=0.01 \mathrm{~m} ; \tau=1800 \mathrm{~s} ;$

$c: w=0.3 \mathrm{~m} / \mathrm{s} ; d_{3}=0.01 \mathrm{~m} ; z_{\mathrm{H}}=0.01 \mathrm{~kg} / \mathrm{m}^{3} ; \tau=1800 \mathrm{~s} ;$

$d: w=0.3 \mathrm{~m} / \mathrm{s} ; \delta_{50}=15 \mu \mathrm{m} ; d_{3}=0.01 \mathrm{~m} ; z_{\mathrm{H}}=0.01 \mathrm{~kg} / \mathrm{m}^{3}$.

- calculated data; - experimental data

It has been experimentally proved that in order to ensure the effective operation of the granular filter, it is necessary to establish rational parameters of its operation, namely: the height of the layer of filter material - in the range of $100 \ldots 150 \mathrm{~mm}$; grain size (average) filling the granular filter in the range of $6.0 \ldots 6.5 \mathrm{~mm}$ with a capacity of up to $200 \mathrm{~m}^{3} / \mathrm{h}$. With a filter capacity of more than $200 \mathrm{~m}^{3} / \mathrm{h}$, its cleaning efficiency is reduced. It has been established that the hydraulic resistance of the developed filter varies from 100 to $900 \mathrm{~Pa}$ with various variable parameters.

\section{Conclussions}

The article proposes a granular filter with a bulk moving layer for cleaning dust from urban roads, in which the filter layer is located stepwise with a decrease in the bulk granular layer. This allowed us to solve 2 main problems when creating filters: eliminating the unevenness of the flow rate of dusty air and reducing the hydraulic resistance of the developed apparatus.

Based on the developed methods for calculating the granular filter, the following dependences were obtained (in a natural and criterial form): 
-Hydraulic resistance of the bulk layer from the main structural and technological factors of the apparatus;

- to predict the hydraulic resistance of the layer through natural variables.

The generalization of the theoretical and experimental studies made it possible to improve granular filters for harvesting machines, which provides an important task in creating a new highly efficient and environmentally reliable urban service technology.

This study was performed with the financial support of the RF Ministry of Education and Science, President Grant \#NSh-3492.2018.8

\section{References}

1. S.Yu. Kabanov, Improving equipment for cleaning dusty gases, Belgorod, BSTU,(2010)

2. A.G. Vetoshkin, Theoretical Foundations of Environmental Protection, Mocscow, Higher school, (2008)

3. Modern filter plants for dust collection in cement factories. Cem. Int. 6 (2003)

4. Rashid Sharapov, Pavel Kapyrin, Svetlana Lozovaya, Valentina Yadykina and Aleksandr Agarkov, Research dedusting efficiency of the inertial hub with adjustable parameters $5^{\text {th }}$ International Scientific Conference "Integration, Partnership and Innovation in Construction Science and Education" 86, (2016)

5. L. S. Brar, R. P. Sharma, and K. Elsayed, "The effect of the cyclone length on the performance of Stairmand high-efficiency cyclone, Pow, Tech. 286, (2015)

6. M. E. Aerov, D. A. Narinsky, Devices with a granular layer. Thermal and hydraulic operation basics. Mocscow, Chemistry, (1979)

7. R.R.Sharapov, Review of the design of granular filters, Belgorod, BSTU, (2009)

8. B.C. Shvydky, M.G. Ladygichev, Gas purification Mocscow, Heat engineer, (2002)

9. R.R. Sharapov, Hydraulic resistance of a granular filter, Belgorod, BSTU, (2011)

10. Rashid R. Sharapov, A.M.Agarkov, Determination of the aerodynamic characteristics of a concentrator with adjustable parameters. MATEC Web of Conferences. $6^{\text {th }}$ International Scientific Conference "Integration, Partnership and Innovation in Construction Science and Education”,(2018)

11. A.M. Agarkov, R.R. Sharapov Mech. of cons. 9. (2016)

12. C. W. Haig, A. Hursthouse, S. Mcilwain et al., "The effect of particle agglomeration and attrition on the separation efficiency of a Stairmand cyclone, Pow. Tech. 258, (2014)

13. A.I. Dotsenko, Utility vehicles and equipment. Mocscow, Architecture-S. (2005).304 p.

14. T.R. Amanbaev, Method for calculating the capture coefficient of particles of a droplet moving in a dusty gas. The, Foun. of Chem.Tech., 42, 3 (2008)

15. V.G. Lisienko, Ya.M. Shchelokov, MG, Ladygichev Equipment of industrial enterprises of the air Reference book: M.: Heat engineer, (2010) 
16. F. Parvaz, S. H. Hosseini, K. Elsayed et al., "Numerical investigation of effects of inner cone on flow field, performance and erosion rate of cyclone separators," Sep. \& Pur. Tech., 201, (2018)

17. T. Zhao, Y. Nakamura, K. Liu et al., "The effect of rolling amplitude and period on particle distribution behavior in a rolling circulating fluidized bed," Pow. Tech. 294, (2016)

18. N.M. Samokhvalov, Cleaning industrial emissions from dust with granular filters, Ec and ind. of Rus. 4, (2009)

19. G.I. Kelbaliev, Resistance coefficients of solid particles, drops and bubbles of various shapes, Theo. Fou. of Ch. Tech. 45, 3 (2011)

20. T. Zhao, J. Yao, K. Liu et al., "Investigation of particle inertial migration in high particle concentration suspension flow by multielectrodes sensing and EulerianLagrangian simulation in a square microchannel, Biomic, 10, 2 (2016) 